become gravely impaired and exhibited signs of failure or impending failure) and as also all convalescents and semiconvalescents were excluded from the treatment. The reasons for such exclusion are sufficiently obvious to all experimenters. Whenever a new mode of treatment has to be tested it is usual to eliminate all disturbing factors that tend to vitiate the final results. And as it was found from our experience of plague that the two above-named classes of cases were such disturbing factors they were excluded. The former when admitted into hospital were generally found to be beyond the reach of all possible human help, and in the case of the latter it was soon recognised that half the battle-nay, more than half and that the most importanthad been already fought and won outside the hospital, and no further assistance was required. Moreover, just as the inclusion of the moribunds would not have told against the serum treatment the non-exclusion of the convalescents would have failed to convince us of its value. Nowbere, so far as I am aware, is such material selected for experiments with new methods of treatment, and in adopting this procedure there was no violent departure from the methods in vogue elsewhere-in fact, there was ample justification for it in a preliminary trial.

The first series of observations lasted from March to November, 1898, and again from February to May, 1899. During the course of this period 403 patients received the serum treatment and 1190 patients were treated by ordinary methods. The clinical effects of the serum were watched from day to day, and although in some very grave and advanced cases the patients ultimately succumbed the immediate effects on the general condition of the patients were extremely well marked, and this indicated either that the dose of the serum was too small, or that it was not strong enough, or that the treatment was commenced too late. But on the whole the improvement was quite perceptible and gratifying. When at the termination of these observations the results were tabulated it was found that the serum-treated cases had a recovery rate of 38.2 per cent. whereas that in those not so treated was 19.5 per cent. only. The latter rate was, however, not less than that of the other large public hospitals, and the combined admissions numbering 4762 in two of the largest during the same period had a recovery rate of 19.7 per cent., and thus it becomes apparent that the exclusion of moribunds and convalescents did not tell in favour of the serum by reducing to any extent whatsoever the mortality rate in those treated by ordinary methods. In fact this system of "selection" so-called or rather mis-called, was so strict and accurate as to eliminate even all mild cases, for were it otherwise the mortality rate in the serum-treated cases ought to have mounted up much higher than the average of the other hospitals.

The second series of observations was commenced in May, 1899 , and is still being continued. The object with which this series was started was to determine the statistical value of the serum, and hence it was resolved to conduct observations on 1000 cases of plague, 500 to be treated with the serum and 500 by ordinary methods, the latter acting as controls, and the cases were to be taken for treatment alternately in the order of admission and without any attempt at cxulusion of ainy sort. But objection to this method of treatment was the preponderance of either moribunds or convalescents in either set vitiating the final results. It was, however, anticipated that in two such large sets of 500 cases each these would equalise at the end, and thus no undue advantage would accrue to one side or the other. We shall see further on whether and to what extent this anticipation was justified by practical experience. Further, in order to arrive at strict accuracy and to eliminate all errors some standard had to be fixed as to what cases should be classed as moribunds and as convalescents. It was therefore determined to fix the limit for the former at 24 hours, and all patients dying within 24 hours of admission were considered as moribunds, but it was subsequently found that a large number died between 24 and 27 hours of admission, and hence 27 hours was adopted as the standard. As regards convalescents, ${ }^{1}$ all those that had gone over the acute stage of the disease and had a normal temperature or thereabouts and had suppurating or suppurated and open buboes were considered convalescent.

And now as tc the results. Up to the end of last month

1 All those that were taken as convalescents in both the serie ultimately recorered, and there was not a single death amongst them.
480 cases have been treated with the serum, and 480 cases have become their controls, under ordinaly treatment:-

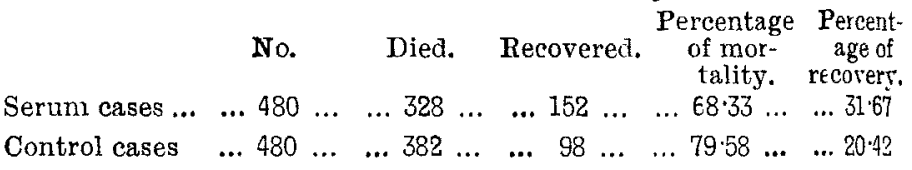

The serum cases show a recovery rate of 3167 per cent., whereas the controls have a rate of 20.42 per cent., a difference in favour of the serum of 1125 per cent. But the above results are not strictly accurate, and bave been rendered fallacious by the undue preporderance of mori. bunds and convalescents in each series. The anticipation at first entertained about the equalising of these has not been realised in actual practice and tbus an element of error bas been introduced into the statistics. The 480 serum cases were found to include 139 moribunds and 28 convalescents, whereas the controls had 145 and 38 respectively. The former had an advantage over the latter of six less mori. bunds, whereas the latter were better off in convalescents by 10. Hence for a strictly accurate comparison, the only course open is to eliminate all the moribunds and all the convalescents from either series, and then deduce results from those remaining. If that is done, the results stand as below.

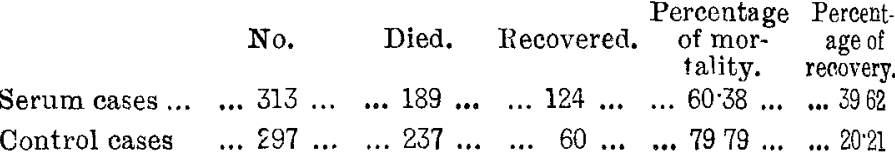

The percentage of recovery stands thus at 39.62 per cent for the serum cases, and 20.21 for the controls, a difference of 19.08 per cent. in favour of serum. These results clearly indicate the value of serum treatment in those cases that are really acute and fit for treatment, and show that in these it is possible to very nearly double the recovery rate by the use of serum. These differences in the percentages do not, however, show the full value of the serum treatment, and that could only be arrived at by following the method adopted by Professor Haffkine in determining the life-saving value of his prophylactic inoculations against plague. That system is based on calculating the relative ratios of mortality amongst the inoculated and non-inoculated, and if the same system be adopted for the serum treatment the ratio of recovery in the serum cases as to control cases would be as 1 of control to 1.55 of serum in all cases, and 1 to 1.96 when the moribunds and convalescents are excluded from both the series. Or to put it in another way, if 100 cases out of a given number recover under ordinary treatment the same number if treated with the serum would give 155 recoveries. And therefore the percentage of recovery is enhanced in one case by 55 per cent and by 96 per cent. in the other.

These, Sirs, are the plain and unvarnished facts with regard to the use of Professor Lustig's serum in the treat. ment of plague and they place before the reader the truth, the whole truth, and nothing but the truth. We have no reason to be dissatisfied with the progress made within two years of its application. Neither the mode of its preparation nor its application at the bedside have yet been finally determined and it is only by further research, experiment, and prolonged clinical observation that we sball be enabled to place it on a proper basis. So far as we have gone we have been able to prove that the serum treatment is the only treatment that can in any way appreciably reduce the high mortality of plague and we abide by our experience. When the diphtheria antitoxin required more than a decade for its perfection is it not reasonable that sufficient time should be allowed for the application of a serum against a much more rapidly fatal and more deadly disease like plague? And is it demanding too much of special correspondents to ask them to witbhold awhile their obiter dicta and let those best conversant with the subject to work out, quietly and undisturbed, their destiny until they reach their goal, which has but one and only aim in viewviz., the lessening of human suffering and the saving of human life?

I am, Sirs, yours faithfully,

N. H. CHOKSY, M.D.
Bombay, July 7th, 1900 .

\section{"RODENT CANCER OF THE CORNEA."} To the Editors of THH LANOET.

SIRs,-The interesting case published under the abore title by Mr. Sydney Stephenson in THE LANCET of July 21st 
would have been much more valuable had the diagnosis of cancer been verified. Judging from Mr. Stephenson's description and diagram the characters of the disease in his case agreed so closely with those of the well-known though somewhat rare chronic creeping ulcer of the cornea, in which there is, I believe, no suspicion of malignancy, that one is compelled to ask in what particulars Mr. Stephenson's case differed from the type? It has always seemed to me unfortunate that the term "rodent," already in use for a form of cancer of the skin, should also have come into use to designate this form of corneal ulcer. If Mr. Stephenson's case were cancerous one can only say that rodent cancer of the cornea, though not hitherto recognised, cannot be very uncommon, for the form of corneal ulcer apparently illustrated by Mr. Stephenson's case is described in most of the standard works on diseases of the eye. The galvanic cautery vigorously applied to the extending border seems to succeed better than any other treatment, but some cases baffle all attempts at cure.

$$
\text { I am, Sirs, yours faithfully, }
$$

$$
\text { July } 25 \mathrm{th}, 19 \mathrm{co} \text {. }
$$

E. NETTLESHIP.

\section{"ARSENIC IN SODIUM PHOSPHATE." \\ To the Editors of THE LANCET.}

SIRS,--In your criticism of the above case contained in your issue of July 21st, p. 208, after stating the fact that we can supply phosphate of soda free from arsenic, you say: "It is a pity, we think, that if, as these druggists state, they knew of the important imparity they did not give greater and earlier publicity to the fact." As manufacturing chemists we certainly do not feel it our duty to warn the public, the medical profession, the wholesale chemists, or other manufacturing chemists of impurities not provided for by the British Pharmacopoia and contained in the product they are interested in. Our duty appears to us to be in making our articles free from these impurities and of the highest standard of purity.

$$
\text { We are, Sirs, yours faithfully, }
$$

MAY AND BAKER, LIMITED

(per W. E. B. Blenkinsop, Managing Director). Battersea, S.W., July 24th, 1900.

\section{"THE COMPARATIVE GERMICIDAL ACTION OF SOME DISINFECTANTS."}

\section{To the Editors of THE LANCET.}

SIRS,-My attention has recently been drawn to a paper under the above heading in THE LANCET of June 23rd (p. 1797), by Mr. Arthur H. Burgess of Manchester, and I write to point out that the comparative results obtained by him are greatly at variance with those of other observers and that in my opinion this discrepancy is due to the method which has been employed in arriving at these conclusions. The thread method was formerly much used, but as far as I am aware is seldom employed now as it was found to give discordant results. In my own practice $I$ bave given it up almost entirely, except in testing spore-bearing organisms under certain conditions and also in experiments on gaseous disinfection, owing to the uncertainty of its results and the difficulties of manipulation.

One of the difficulties attending the thread method is that doe to grease, and Mr. Burgess in his paper does not mention whether he took precautions to ensure the freedom of the threads from grease, as he only refers to sterilising them before use. The most important error, in my opinion, arises from the fact that when the threads are partially dried withont any attempt being made to remove the organic matter of the broth emulsion, a film of dry albuminous matter is formed which protects the organisms, and that therefore I cannot agree with Mr. Burgess when he says that "the bacilli are freely exposed to the action of the disinfectant in solution, no albuminous matter being present." Another point which is worth bearing in oind is the fact that it is almost impossible to condoct a series of inoculations in such a short interval as one minute, and if $\mathrm{Mr}$. Burgess used different cultures for his one-minute experiments then the results are not strictly comparable. I notice that his sub-cultures were incubated for 10 days. This time seems unduly prolonged, as with most cultures of bacillus coli communis a growth or tarbidity occurs in a much shorter time than that when kept at blood heat. The method described by Mr. J. T. A. Walker in his letter ${ }^{l}$ is one which I have employed for some years in a slightly modified form, and I believe it to be at present that which gives the most regular and constant results, and it would be interesting if Mr. Walker would publish in your journal the experiments referred to in his letter as they could then be compared with the results obtained by $\mathrm{Mr}$. Burgess. I am, Sirs, yours faithfully,

SAMUEL RIDEAL.

Victoria-mansions, Westminster, S.W., July 25th, 1900.

\section{PLAGUE IN "TURKEY.}

(FROM A CORRESPONDENT.)

For some months past plague has been present in more than one portion of the Turkish Empire, but up to the present it has nowhere given rise to anything of the nature of a severe epidemic. The outbreak which has naturally caused the greatest apprehension has been that in Smyrna, on account of the size and importance of the town, of the amount of shipping constantly visiting the port, and of its proximity to Constantinople. Fortunately, the outbreak has so far been of a very mild character, only 17 cases in all having been reported in the course of over two months. But the slowness with which plague takes hold of a place is a well-known character of the disease, and bearing the example of Calcutta and other places in mind it is impossible not to feel some degree of apprehensiveness for the future behaviour of plague in Smyrna.

The first recognised case of the disease there was seen on May 7th, when the patient had already been ill some few days. The patient was an elderly Jew, 60 years of age, living apparently in very poor circumstances. The second case occurred on May 26th, or nineteen days after the recognition of the first case. This patient was also a Jew, a youth, aged 23 years, who occupied a than some quarter of a mile away from that occupied by the first patient; he was taken ill apparently on May 25th. Another short interval without a case followed, but on the night of June 4th-5th a third person fell ill with the disease. This was a boy, an Ottoman, aged 14 years, acting as domestic servant in the SkiTchesmelik quarter, a long way from the part of the town where the other patients had lived. The fourth case was also that of a Turk, a man aged 45 years, who lived outside the town of Smyrna, on a hill called Mount Bech-Tepé, where a few people dwell in a small group of isolated huts. He was first seen on June 15th, but the date of his attack is uncertain. All these four persons suffered from the disease in a remarkably mild form and all recovered. In each the diagnosis was made certain by the isolation of the plague bacillus from the bubo. In none could any direct connexion with a previous case of the disease be traced. The fifth case, on the other hand, ended in death, and it proved to be the first of a group of cases which occurred in and around a particular khan or group of houses. This is known as the Echeref-Pasha khan and is situated in the Bach-Durak quarter of the town. The fifth, sixth, seventh, ninth, and eleventh cases of plague were all in persons inhabiting this khan or its neighbourhood. The remaining cases, into the details of which it is scarcely necessary to enter, occurred in various other quarters of the town, and little or no trace of connexion could be found between the successive cases. The last was reported on July 8th; the patient had been ill since the 5th. A week has now elapsed without a fresh case being reported; this is reassuring as far as it goes, but in view of the wide extension of the infection throughout the town of Smyrna only the most sanguine can venture to hope that the outbreak is over and the infection completely eradicated. A less reassuring feature of the outbreak is the tendency shown to a successive increase in the fatality of the disease. At the ontset it was of a mild character, and the first four cases all recovered, as already stated. Then followed, between June 17 th and $27 \mathrm{th}$, ten cases, of which three proved fatal ; but on July 1st, 4th, and 8th three fresh cases were reported, every one of which proved fatal. It is interesting, in connexion wilh the Smyrma outbreak, that no sickness or mortality among rats has, it 